\title{
Using 3D printed sinonasal models to visualize and optimize personalized sinonasal sinus irrigation strategies*
}

\author{
Kai Zhao', Kanghyun Kim', John R. Craig², James N. Palmer ${ }^{3}$ \\ 'Department of Otolaryngology- Head \& Neck Surgery, The Ohio State University, Columbus, OH, USA \\ 2Department of Otolaryngology- Head \& Neck Surgery, Henry Ford Health System, Detroit, MI, USA \\ ${ }^{3}$ Department of Otolaryngology- Head \& Neck Surgery, Perelmann School of Medicine at University of Pennsylvania, Philadelphia, \\ $\mathrm{PA}$, USA
}

Rhinology 58: 0, 0 - 0, 2020

https://doi.org/10.4193/Rhin19.314

September 11, 2019

Accepted: December 15, 2019

\begin{abstract}
Background: Topical sinus irrigations (neti-pot, squeeze bottles) play a critical role in the management of sinonasal disease. However, due to intricate nasal anatomy, penetration of topical irrigations to targeted sinus regions may be highly variable, and difficult to objectively predict. Variables, including head positions, injection angles, flow rates, etc. may vary significantly depending on the individual's anatomy.
\end{abstract}

Objective: The purpose of this study was to propose a novel idea: using a 3D printed model of sinonasal cavities to visualize and develop a patient-specific irrigation strategy.

Methods: As a proof of concept, 3D replicas of one patient's sinonasal cavities pre- and post-surgery were printed with a Form2 SLA 3D printer based on their CT scans. The setup included rubber/silicon seals attached to the model's nostrils to create a watertight seal with the irrigation device and food color dye added for better visualization of irrigation results.

Results: Irrigations were performed on the 3D models with various head positions, injection angles, and flow rates, and were successful to determine the optimal strategy to targeted sinuses. Significant differences were observed between different targeted sinuses and between pre and post-surgery models.

Conclusion: With more affordable 3D printing, this technology may potentially improve patient care and patient education, allowing clinicians and patients to develop a personalized irrigation strategy and have visual confirmation.

Key words: topical sinus irrigation, 3D printing modeling, sinusitis, maxillary, head position

\section{Introduction}

Chronic rhinosinusitis (CRS) is one of the most common chronic medical conditions in the US, affecting an estimated $13 \%$ of adults, or some 30 million people (National Health Interview Survey 2009, CDC). Topical therapies play an integral role in the management of CRS, and high-volume irrigation delivery (e.g., neti pot, squeeze bottles) is more effective for achieving distribution to the sinuses than other topical delivery methods such as nasal sprays, nebulizers, or atomizers ${ }^{(1-5)}$. Saline irrigations have been recommended in a number of clinical scenarios, including initial management of CRS ${ }^{(6)}$ and postoperative care (7). High-volume irrigations have also shown benefits for medi- cation delivery, such as with mupirocin (8) and corticosteroids $(3,9,10)$. However, due to the intricate and variable anatomy of the human nasal airway, the efficacy of topical irrigations to reach targeted sinuses is inconsistent and difficult to predict. Previous studies have shown that nasal irrigants may not reliably penetrate all sinuses ${ }^{(11)}$, and the effectiveness depends on specific sinuses, head positions, injection angle, flow rates, and individual anatomy ${ }^{(12)}$. We currently do not have a clear understanding on the combination of settings for optimal topical delivery. Investigations into determining the distribution of irrigations within the sinuses have been limited by labor-intensive methodologies, such as cadaver studies or using colored dyes 
under endoscopic view ${ }^{(11,12)}$. Other studies have used irrigations with iodinated contrast followed by computed tomography (CT) scans to determine which sinuses collect contrast material ${ }^{(2,10)}$. Similarly, ${ }^{99 m}$-technetium sulfur colloid ${ }^{(1)}$ and fluorescein ${ }^{(13)}$ labeled irrigations have also been used as tracers to determine the distribution of sinus irrigations. These labor-intensive techniques with increased patient risk are difficult to apply to the general patient population. More recently computational fluid dynamics (CFD) has been used to simulate sinonasal irrigations (14-16), which has the following advantages over other methods: less labor-intensive, requiring only a CT scan, capturing the complete dynamic moment-to-moment irrigation paths, and can be patient-specific but cause no discomfort to the patient. However, a drawback of CFD is the need for intensive computational resources, which can takes weeks to complete, even with parallel processing through multicore CPU or computer clusters.

From both patients' and clinicians' perspectives, the lack of patient-specific irrigation prediction can be frustrating, as clinicians may prescribe a rigorous irrigation routine but cannot be certain patients are performing them effectively. When symptoms fail to improve after topical medication delivery via irrigation, it is difficult to determine whether the irrigationdelivered medication failed, or the irrigation did not reach the clinically relevant sinus cavities. Many patients and surgeons then opt for systemic medication or surgery, which increases risk either from medication side effects, or the various risks incurred during surgery.

The purpose of this study was to propose a novel idea: applying three-dimensional (3D)-printing technology based on individual patient's computed tomography (CT) scans to determine an optimal personalized nasal irrigation strategy (head positions, angle of injection, flow rates, etc.). 3D printing technology is an additive manufacturing process. 3D printing techniques has been previously used in the field of otolaryngology for better visualizing the nasal sinus anatomy and for surgical training ${ }^{(17-20)}$, however, to date, it has not been reported for study or for optimization of sinonasal irrigation distribution.

\section{Material and methods}

A patient's CT scan can be converted into a sinonasal model with 3D patient specific features using computer-aided design software. First, the interface between the nasal mucosa and the air was delineated on the $C T$ scans using an image processing software (AMIRA, Visualization Sciences Group, Burlington, MA, USA), a process that can be done with other imaging software following the same principle. Then, a $\sim 3 \mathrm{~mm}$ thick wall was created to enclose the nasal air space (Figure 1b). The thickness of the wall is chosen to both structurally support the printed model and provide a clear view. A thinner wall will make the
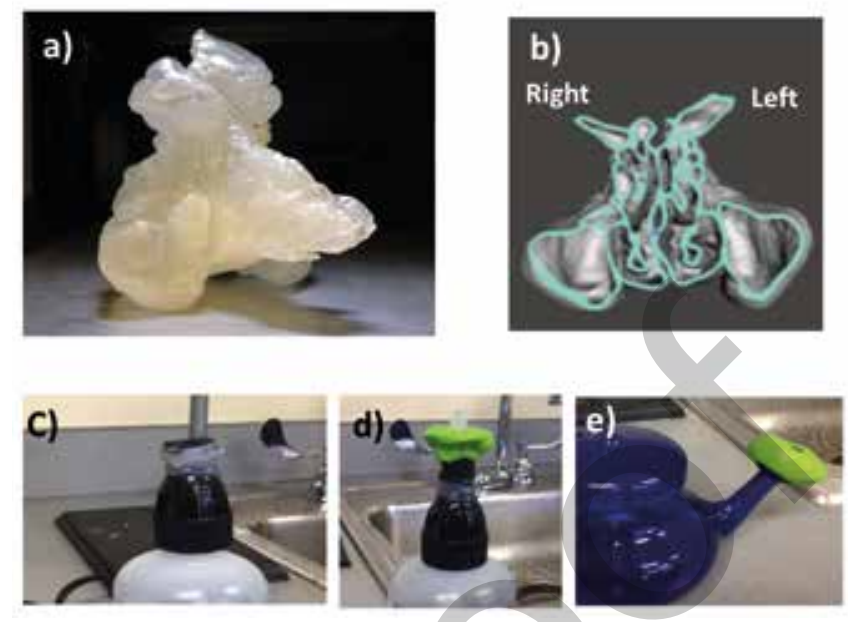

Figure 1. a) a 3D printed nasal replica based on one patient's specific CT scan. b) a cross-section of the 3D printed nasal replica. A wall of $3-4 \mathrm{~mm}$ thickness is created to enclose the nasal air space. c-e) various attachment of water-tightly connection for common irrigation devices to the 3D printed nasal model. As examples, we have (c) rubber molds hot glued onto a squeeze bottle, (d) silicon deformable and detachable molds on a squeeze bottle, (e) similar silicon molds on a typical net-pot. They all serve the similar function of creating a water-tight connection between the irrigation device and the nasal replica so that irrigation trials can be easily performed over a sink, as shown in Figure 2, 3 and Video 1.

model too fragile and a thicker wall will make the model less transparent. However, the wall is specially designed not to encroach into the nasal or sinus airspace, nor disrupt the thin bony walls at various delicate regions. Both nostrils were left open, into which the irrigation device tip could be inserted, and through which irrigation could be forced at different flow rates. Both of the nostril planes were specially treated to be flat and smooth to allow for better water-tight sealing with the delivery attachment. The contralateral nostril with respect to the nostril used as the inlet will serve as an outlet (the only outlet), through which air and irrigant could exit. The nasopharyngeal opening was blocked off and impenetrable to liquid or air, representing the closure of the soft palate. Full closure of the soft palate in true life is only possible during a short period, while swallowing, sustained breath holding or pronouncing the consonant " $\mathrm{k}$ " repeatedly. Our assumption is based on the fact that most patients performing nasal sinuses irrigation while breath-holding and is concerned about fluid entering pharynx airway. It remains to be investigated whether closure of the soft palate would still be needed in e.g. a $90^{\circ}$ prone head position. The final 3D nasal digital model was then saved as a STL file and a 3D printer was used to print material into a suitably designed sinonasal replica.

As proof of concept, 3D exact replicas of one patient's sinonasal 
cavities before and after surgery were printed with a Formlabs Form 2 Stereolithography (SLA) 3D printer based on the aforementioned methods (Figure 1a). We used a SLA 3D Printer, as it can print semi-transparent material. Other 3D printing technologies could also be suitable for creating these sinonasal models, following the same principle. This patient was a 47 year-old male with chronic rhinosinusitis who had undergone revision complete endoscopic sinus surgery. The surgery included bilateral wide maxillary antrostomies, total ethmoidectomies, sphenoidotomies, and a Draf III frontal sinusotomy. The Draf III procedure included resection of the anterosuperior portion of the middle turbinates, a superior nasal septectomy, and drilling out of the nasofrontal beak and frontal intersinus septum.

Another key aspect of the proposed idea was the designing of a water-tight connection of the nasal replica to various common irrigation devices. Figure 1 (C-E) showed various examples: (c) rubber molds hot glued onto a squeeze bottle, (d) silicon deformable and detachable molds on a squeeze bottle, (e) similar silicon molds on a typical net-pot. They all served the similar function of a water-tight connection between the irrigation device and the nasal replica, so that irrigation trials could be easily performed over a sink, as shown in Figures 2 and 3. Colored food dye was also added to the water for better visualization of irrigations.

\section{Results}

As proof of concept, we performed irrigation trials on the two $3 \mathrm{D}$ nasal replicas with various head positions and irrigation devices to demonstrate that it can be easily used to determine optimal irrigation settings for better outcome. Each nasal replica was clamped on a steel chemistry support stand, with its orientation angle calibrated by a laser angular protractor. The following five head positions were studied using a squeeze bottle to irrigate (Supplementary Figure 1): a) $45^{\circ} \mathrm{Head}$ Tilt to Left (in coronal plane), b) $90^{\circ}$ Head Tilt to Left (in coronal plane), c) $45^{\circ}$ Head Tilt to Left (coronal) and $45^{\circ}$ Head Tilt Forward (in horizontal plane), d) $45^{\circ}$ Head Tilt Forward (in horizontal plane), e) $90^{\circ}$ Head Tilt Forward (in horizontal plane). As shown in Video 1, each irrigation trial only took a few seconds, so it is easy to replicate with various other angles and irrigation settings. But due to video and manuscript length limitations, not all of the angles and settings could be presented. Nevertheless, one can still appreciate the significant variations in terms of irrigation outcomes, even within these limited head positions.

Figure 2 depicts irrigation distribution for the preoperative model at different head positions (Video1 for full trials). Figure 2a) compared irrigation delivery to the maxillary sinus, which showed that except for the $45^{\circ}$ Head Tilt to Left, none of the other head positions resulted in any visible filling of the maxil- lary sinus. Figure $2 \mathrm{~b}$ ) compared the results for ethmoid sinus delivery, in which $90^{\circ}$ Head Tilt to the side demonstrated the best outcome. Figure 2c) highlighted frontal sinus delivery, where the $90^{\circ}$ Head Tilt Forward demonstrated the best outcome. One key point was that results were highly variable depending on head position, and certain sinuses could only be reached in certain head positions.

Figure 3 showed irrigation distribution results in the postoperative model, which in general was much improved compared to the preoperative state, though there were still significant variations depending on different head positions. For example, in Figure 3a), both $45^{\circ}$ Head Tilt to Left and $90^{\circ}$ Head Tilt to Left showed completely full filling of the contralateral maxillary sinus, whereas the $45^{\circ}$ Head Tilt Forward only received a light filling. But since $45^{\circ}$ Head Tilt to Left even received half filling of the ipsilateral maxillary sinus, it was considered an overall better position than the $90^{\circ}$ Head Tilt to Left for maxillary sinuses. In Figure $3 \mathrm{~b}$, only $90^{\circ}$ Head Tilt to Left showed moderate filling of ethmoid and sphenoid sinus, whereas none of the other head positions received any visible filling of ethmoid and sphenoid sinuses. In Figure 3c, even though the $90^{\circ}$ Head Tilt Forward received full frontal sinuses filling, there was no penetration of any of the other sinuses; whereas the $90^{\circ}$ Head Tilt to the side position actually received irrigation of the majority of the frontal sinus as well as irrigation of the contralateral maxillary and ethmoid sinuses. There could be a split of opinion, but based on overall performance, the $90^{\circ} \mathrm{Head}$ Tilt to the side position could be the optimal position for all sinuses for this patient's postsurgery sinonasal cavities.

There were some general agreements for this patient's most optimal head position pre- and postoperatively. For example, the $45^{\circ}$ Head Tilt to Left was generally good for maxillary sinus irrigation; the $90^{\circ}$ Head Tilt to Left was generally good for ethmoid and sphenoid sinuses; the $90^{\circ}$ Head Tilt forward was generally good for frontal sinuses; the $45^{\circ}$ Head Tilt Forward was a poor position for almost all sinuses. But there were still many discrepancies, and there were positions that the postoperative model resulted in worse irrigation distribution than the preoperative model. For example, the $90^{\circ}$ Head forward position received light filling of the ethmoid sinuses in the preoperative model, but there was no visible filling in the postoperative model. Other variations were seen as well: the $90^{\circ}$ Head Tilt to Left resulted in complete maxillary sinus filling postoperatively, but there was no visible filling preoperatively.

\section{Discussion}

CRS significantly impacts patient quality of life and affects sleep/ productivity-related outcomes. There is evidence that if sufficient drug or irrigation can be appropriately delivered to the 
Figure 2. displayed the snapshot of irrigation fluid level for the pre-surgery model at different head positions (see Video1 for full trials). a) specifically compared the irrigation outcome to the maxillary sinus, which showed that except for the $45^{\circ}$ Head Tilt to Left, none of the other head positions resulted in any visible filling of the maxillary sinus. b) summarized the ethmoid sinus, in which $90^{\circ}$ Head Tilt to the side demonstrated the best outcome. c) showed the frontal sinuses, where the $90^{\circ}$ Head Tilt Forward demonstrated the best outcome. In brief conclusion, the optimal head positions for different sinuses can be very different.
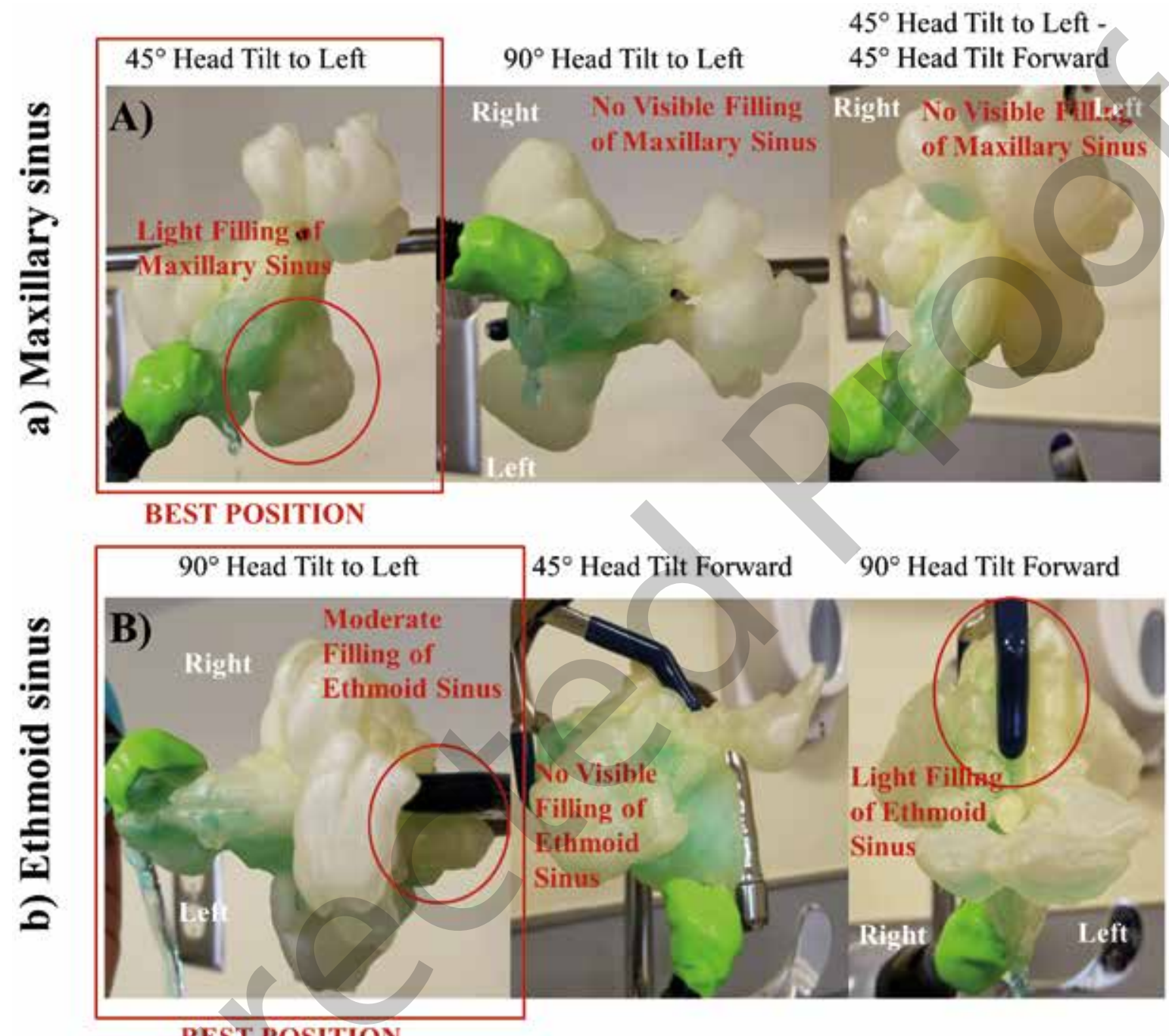

BEST POSITION

$45^{\circ}$ Head Tilt to Left $45^{\circ}$ Head Tilt Forward

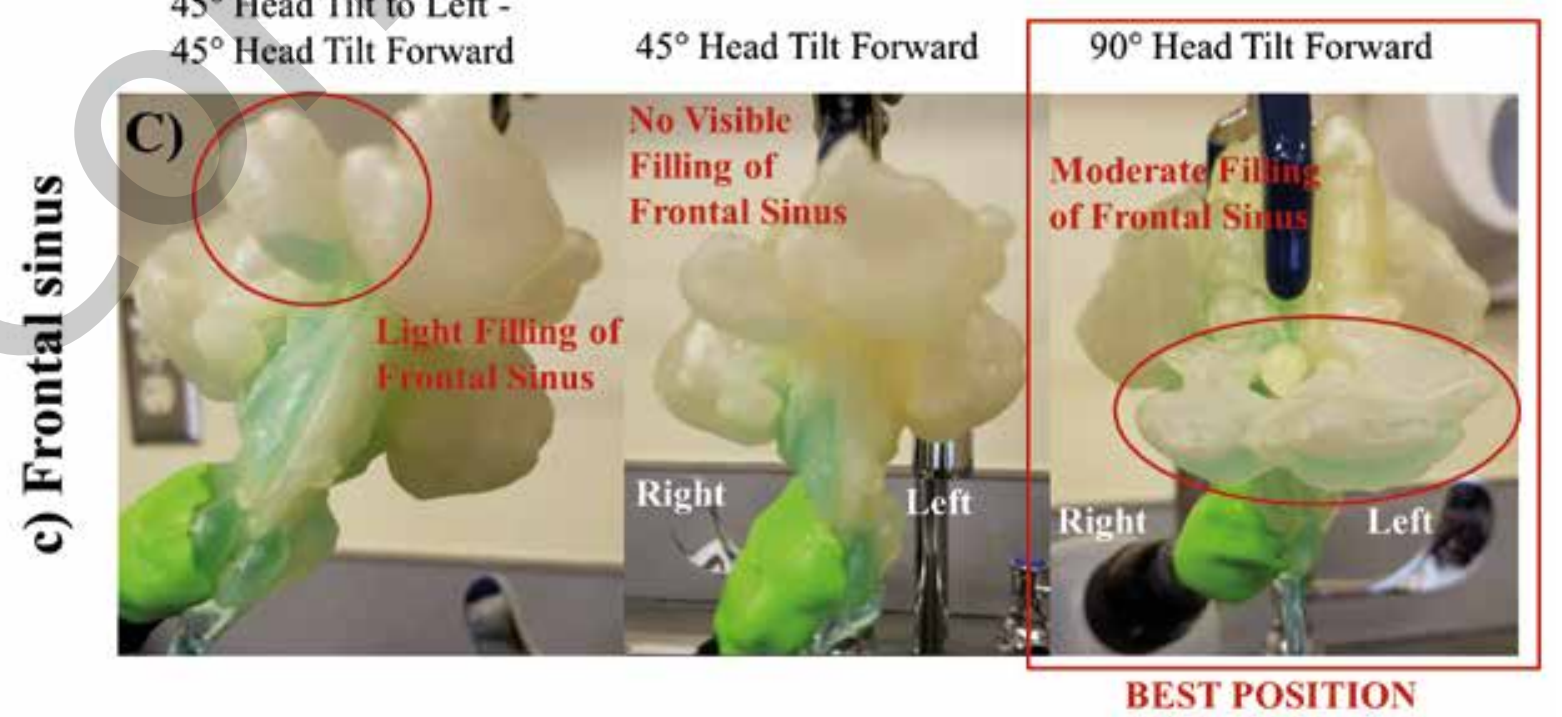


Figure 3. The fluid filling level for the post-surgery model is shown, which in general was much improved compared to the pre-surgery case, but still with significant variations for different head positions or different targeted sinuses. For example, a) both $45^{\circ}$ Head Tilt to Left and $90^{\circ} \mathrm{Head}$ Tilt to Left received completely full filling of the contralateral maxillary sinus, whereas $45^{\circ} \mathrm{Head}$ Tilt Forward only received a light filling. But since $45^{\circ} \mathrm{Head}$ Tilt to Left even received half filling of the ipsilateral maxillary sinus, it is probably a better position than the $90^{\circ}$ Head Tilt to Left for maxillary sinuses. b) only $90^{\circ}$ Head Tilt to Left received moderate filling of ethmoid and sphenoid sinus, whereas none of the other head positions received any visible filling of ethmoid and sphenoid sinuses. c) while the $90^{\circ}$ Head Tilt Forward received full frontal sinuses filling, none of the other sinuses received penetration; whereas the $90^{\circ} \mathrm{Head}$ Tilt to the side position actually received irrigation of the majority of frontal sinus as well as receiving irrigation of the contralateral maxillary and ethmoid sinuses. There could be a split of opinion, but based on overall performance, the $90^{\circ}$ Head Tilt to the side position could be the optimal position for all sinuses for the post-surgery case.
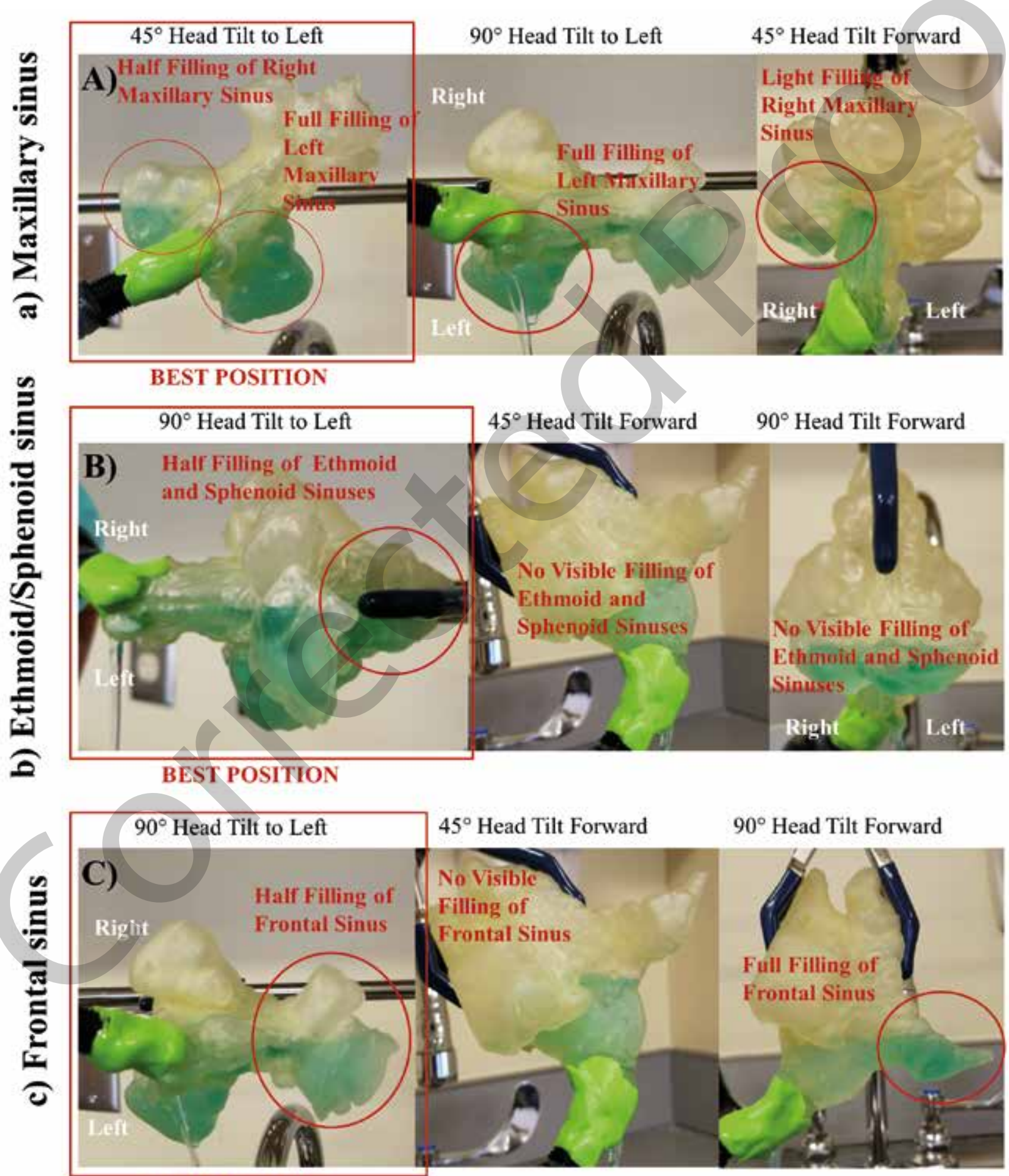

BEST POSITION 
targeted sinuses, majority of CRS symptoms can potentially be improved, and can reduce the need for surgery and systematic medication ${ }^{(3)}$. The use of 3D printing to optimize sinus irrigation outcomes has the potential to directly translate from research to clinical practice. Results from this proof-of-concept study showed that significant variability existed in irrigation distribution outcomes, depending on the anatomy and head position, even in the same patient pre- versus postoperatively. By applying this new application of 3D printing technology, sinonasal irrigation drug delivery could potentially be optimized through iterative processes to account for individual patient's anatomy (e.g. septal deviation or not) and to maximize treatment outcomes. This personalized-medicine approach may incorporate both physician and patient involvement.

Physicians may utilize the 3D printed model as an educational tool, pointing out sinus cavities to the patient and marking where the most diseased regions are for a particular patient. Patients can then use 3D printed models to practice the recommendation irrigation dosage and develop their own personal irrigation strategy over the sink at home and have visual confirmation of irrigation penetration to the diseased region. This would be valuable even for patients in rural areas or in disadvantaged communities without access to 3D printing technology; they could potentially mail in their $\mathrm{CT}$ to a centralized center and have their 3D-printed nasal replica mailed back. The 3Dprinted model may also serve as an important patient education tool to improve patient compliance. One problem with nasal irrigation is patient compliance, since the irrigation is not an entirely pleasant experience. With visual confirmation, patients may have increased confidence and motivation to follow through with daily prescribed irrigation routines. The 3D-printed model may also educate patients on the importance of the precision of head angles. Griggs et al. ${ }^{(21)}$ showed that patients in a rhinology clinic irrigated on average about $20^{\circ}$ up-rotated on lateral view, and vertex rotated $10^{\circ}$ away from the side of irrigation on frontal view. As was shown in the current study, head positions are not all created equal, and perhaps with a 3D printed model, patients would be more likely to irrigate in a more precise manner since they know an error will not result in maximal sinus delivery.

Optimizing topical irrigation outcome is an important area of research; utilizing 3D printing will also provide much-needed clinical insight into how to decide optimal irrigation parameters based on disease types and targets within a general patient population. On an individual basis, analysis of irrigant flow using the 3D printed models also has the potential to provide insight into what surgical maneuvers may optimize sinonasal drug delivery and act as a guide to improve surgical outcome in the future. Limitations of this paper obviously include that this is a proof of concept study, and would require a larger sample size study to define how much the 3D-printed model would improve personalized clinical decision and outcome, which we are in the process of conducting. Additionally, in order to fully establish the value of the 3D-printed models in patient education and compliance, assessments of patient reception of the technology may be required, which are included in our next phase of the project.

\section{Conclusion}

The development of a 3D printing clinical tool could offer great promise to improve nasal sinus irrigation outcome, and to potentially improve patient care based on objective evidence and personalized medicine approach.

\section{Acknowledgements}

This research was supported by NIH NIDCD R01 DC013626 to KZ.

\section{Authorship contribution}

$\mathrm{KZ}$ conceptualized the idea, KZ and KK designed the study, KK and $K Z$ performed the study and data analysis, JC and JP provide clinical insight, KZ JC and JP wrote the manuscript.

\section{Conflict of interest}

A US patent is pending. The authors have no other financial interest and conflict of interest to disclose.

\section{References}

1. Wormald P-J, Cain T, Oates L, Hawke $L$, Wong I. A comparative study of three methods of nasal irrigation. Laryngoscope. 2004;114(12):2224-2227.

2. Harvey RJ, Goddard JC, Wise SK, Schlosser RJ. Effects of endoscopic sinus surgery and delivery device on cadaver sinus irrigation. Otolaryngol--Head Neck Surg Off J Am Acad Otolaryngol-Head Neck Surg. 2008;139(1):137-142.

3. Harvey RJ, Snidvongs K, Kalish LH, Oakley GM, Sacks R. Corticosteroid nasal irrigations are more effective than simple sprays in a randomized double-blinded placebocontrolled trial for chronic rhinosinusitis after sinus surgery. Int Forum Allergy Rhinol. 2018;8(4):461-470.

4. Thomas WW, Harvey RJ, Rudmik L, Hwang $\mathrm{PH}$, Schlosser RJ. Distribution of topical agents to the paranasal sinuses: an evidence-based review with recommendations. Int Forum Allergy Rhinol. 2013;3(9):691-703.

5. Doellman M, Chen PG, McMains KC, Sarber KM, Weitzel EK. Sinus penetration of saline solution irrigation and atomizer in a cadaveric polyp and allergic fungal sinusitis model. Allergy Rhinol Provid RI. 2015;6(1):811.

6. Achilles N, Mösges R. Nasal saline irrigations for the symptoms of acute and chronic rhinosinusitis. Curr Allergy Asthma Rep. 2013;13(2):229-235.

7. Liang K-L, Su M-C, Tseng H-C, Jiang R-S. Impact of pulsatile nasal irrigation on the prognosis of functional endoscopic sinus surgery. J Otolaryngol - Head Neck Surg J Oto-Rhino-Laryngol Chir Cervico-Faciale. 2008;37(2):148-153.

8. Jervis-Bardy J, Boase S, Psaltis A, Foreman A, Wormald P-J. A randomized trial of 
mupirocin sinonasal rinses versus saline in surgically recalcitrant staphylococcal chronic rhinosinusitis. Laryngoscope. 2012;122(10):2148-2153.

9. Jang DW, Lachanas VA, Segel J, Kountakis SE. Budesonide nasal irrigations in the postoperative management of chronic rhinosinusitis. Int Forum Allergy Rhinol. 2013;3(9):708-711.

10. Snidvongs $K$, Kalish $L$, Sacks $R$ Sivasubramaniam R, Cope D, Harvey RJ. Sinus surgery and delivery method influence the effectiveness of topical corticosteroids for chronic rhinosinusitis: systematic review and meta-analysis. Am J Rhinol Allergy. 2013;27(3):221-233.

11. Grobler A, Weitzel EK, Buele A, et al. Pre- and postoperative sinus penetration of nasal irrigation. Laryngoscope. 2008;118(11):20782081.

12. Singhal D, Weitzel EK, Lin E, et al. Effect of head position and surgical dissection on sinus irrigant penetration in cadavers. Laryngoscope. 2010;120(12):2528-2531.

13. Bleier BS, Preena D, Schlosser RJ, Harvey RJ. Dose quantification of topical drug delivery to the paranasal sinuses by fluorescein luminosity calculation. Int Forum Allergy Rhinol. 2012;2(4):316-320.

14. Zhao K, Craig JR, Cohen NA, Adappa ND, Khalili S, Palmer JN. Sinus irrigations before and after surgery-Visualization through computational fluid dynamics simulations. Laryngoscope. 2016;126(3):E90-96.

15. Craig JR, Zhao K, Doan N, et al. Cadaveric validation study of computational fluid dynamics model of sinus irrigations before and after sinus surgery: CFD sinus irrigation model. Int Forum Allergy Rhinol. 2016;6(4):423-428.

16. Craig JR, Palmer JN, Zhao K. Computational fluid dynamic modeling of nose-to-ceiling head positioning for sphenoid sinus irrigation. Int Forum Allergy Rhinol. 2017;7(5):474-479.

17. Waran V, Menon R, Pancharatnam D, et al. The creation and verification of cranial models using three-dimensional rapid prototyping technology in field of transnasal sphenoid endoscopy. Am J Rhinol Allergy. 2012;26(5):132-136.

18. Chang DR, Lin RP, Bowe S, et al. Fabrication and validation of a low-cost, mediumfidelity silicone injection molded endoscopic sinus surgery simulation model. Laryngoscope. 2017;127(4):781-786.

19. 19. Alrasheed AS, Nguyen LHP, Mongeau L, Funnell WRJ, Tewfik MA. Development and validation of a 3D-printed model of the ostiomeatal complex and frontal sinus for endoscopic sinus surgery training. Int Forum Allergy Rhinol. 2017;7(8):837-841.

20. Crafts TD, Ellsperman SE, Wannemuehler TJ, Bellicchi TD, Shipchandler TZ, Mantravadi
AV. Three-Dimensional Printing and Its Applications in Otorhinolaryngology-Head and Neck Surgery. Otolaryngol--Head Neck Surg 2017;156(6):999-1010.

21. Griggs ZH, M Williams A, Craig JR. Head and Bottle Angles Achieved by Patients During High-Volume Sinonasal Irrigations. Am J Rhinol Allergy. 2019:1945892419825612.

Kai Zhao, Ph.D., Associate Professor Department of Otolaryngology

Head \& Neck Surgery

The Ohio State University

915 Olentangy River Rd.

Office 4235

Columbus 43212, OH

USA

Tel: +1-614-293-3857 (office); +1-614-

366-1794 (lab);

Fax: +1-614-293-7476,

E-mail: zhao.1949@osu.edu

This paper contains supplementary information (video and figure) online: at www.rhinologyjournal.org 


\section{SUPPLEMENTARY INFORMATION}

Video 1 shows how a patient can easily perform and practice irrigation trials on the replica of their own noses over a sink with the water-tight attachment (shown in Figure S1), develop their own personal optimal irrigation strategy (head position, irrigation flow rate, irrigation devices, etc.) and have visual confirmation at home.

$45^{\circ}$ Head Tilt to Left

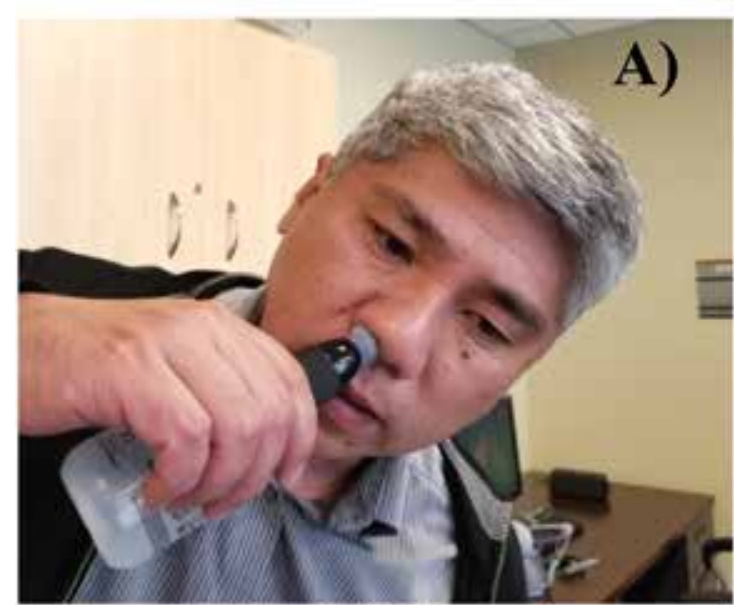

$90^{\circ}$ Head Tilt Forward

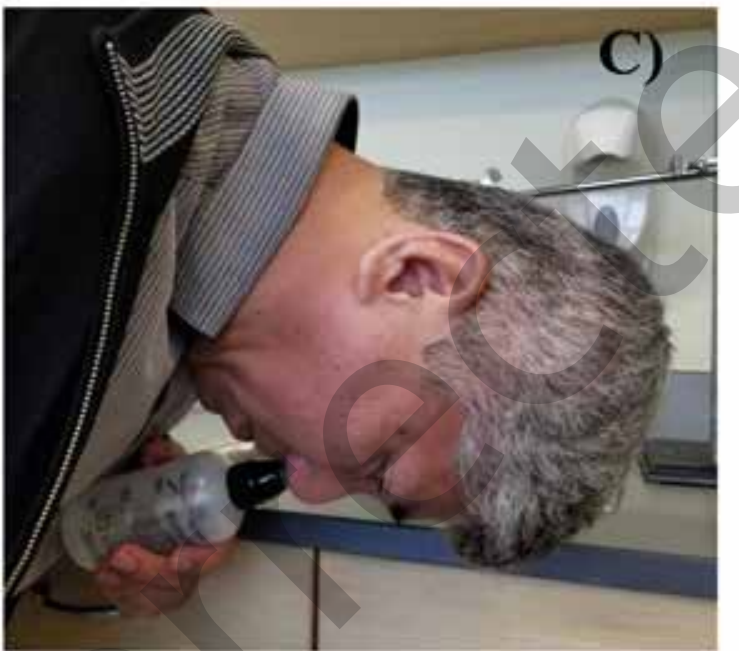

$45^{\circ}$ Head Tilt to Left $-45^{\circ}$ Head Tilt Forward

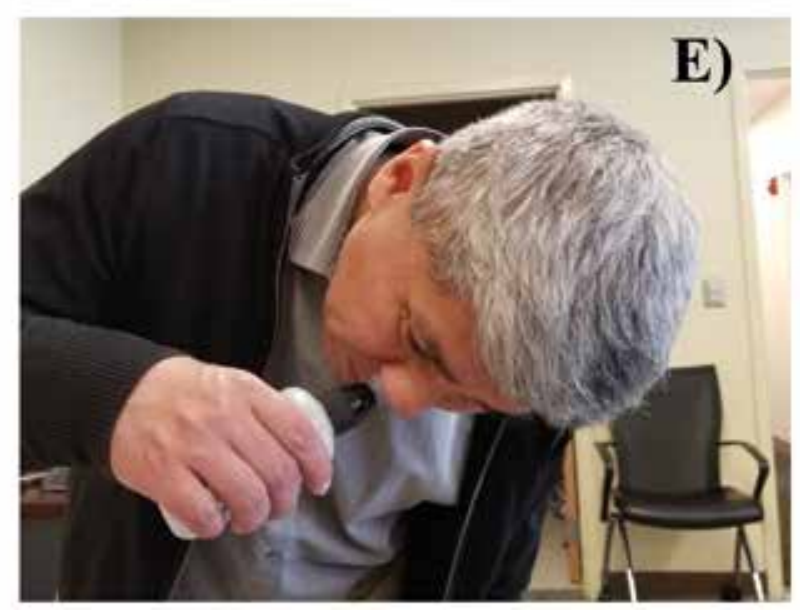

